\title{
Phase synchronization and polarization ordering of globally coupled oscillators
}

\author{
Alessandro Scirè, ${ }^{*}$ Pere Colet, ${ }^{\dagger}$ and Maxi San Miguel $^{\ddagger}$ \\ Instituto Mediterráneo de Estudios Avanzados, IMEDEA (CSIC-UIB), Campus Universitat Illes Balears, \\ E-07122, Palma de Mallorca, Spain
}

(Received 23 March 2004; published 8 September 2004)

\begin{abstract}
We introduce a prototype model for globally coupled oscillators in which each element is given an oscillation frequency and a preferential oscillation direction (polarization), both randomly distributed. We found two collective transitions: to phase synchronization and to polarization ordering. Introducing a global-phase and polarization order parameters, we show that the transition to global-phase synchrony is found when the coupling overcomes a critical value and that polarization order enhancement cannot take place before globalphase synchrony. We develop a self-consistent theory to determine both order parameters in good agreement with numerical results.
\end{abstract}

DOI: 10.1103/PhysRevE.70.035201

In recent years a considerable interest has been devoted to the self-organization properties exhibited by networks of coupled nonlinear oscillators [1]. The work of Winfree [2] first showed that the study of self-sustained non-identical oscillators is a suitable framework to achieve insight into the synchronization processes in biological systems. Based on Winfree's approach, Kuramoto [3] proposed a treatable model for synchronizing oscillators successfully exploited in many fields, from heart physiology [4] to superconducting junctions [5]. The underlying idea behind this success is that in many instances the dynamics of the individual oscillators can effectively be described as a limit cycle in which only one phase plays a relevant role. Then, for small disorder and weak coupling the Kuramoto model provides an excellent description of the synchronization process. A limitation of this model is that it does not consider the possible different direction of oscillation of the coupled oscillators. Whereas, for instance, the Kuramoto model has been generalized including inertial effects [6], the relationship between phase synchronization and a possible collective ordering of the oscillation direction has not yet been addressed. Such general question would arise, for example, when considering coupled oscillators, each of which can oscillate in an arbitrary direction in a plane. This is of direct relevance in the field of optics: the cooperative behavior encountered in laser arrays has been investigated both from experimental $[7,8]$ and theoretical [7,9] points of view including descriptions in terms of the Kuramoto model [10] where the global coupling arises from light feedback from an external mirror. However, the vectorial nature of the electric field imposes a fundamental limitation to the description in terms of single phase oscillators. This description can only be used when the polarization degree of freedom is completely fixed by natural constraints. This is not the case, for example, in arrays of vertical-cavity

\footnotetext{
*Electronic address: scire@imedea.uib.es; URL: http://www.imedea.uib.es/PhysDept

†Electronic address: pere@imedea.uib.es; URL: http://www.imedea.uib.es/PhysDept

*Electronic address: maxi@imedea.uib.es; URL: http://www.imedea.uib.es/PhysDept
}

PACS number(s): 82.40.Bj, 42.60.Da, 05.45.Xt, 42.25.Ja

surface-emitting lasers (VCSELs) [11], where the polarization of the emitted light is not fixed by the structure [12], and the interplay between polarization and electric field globalphase requires at least a description in terms of two phases for each element. Indeed, it is possible to have states in which the global phases are synchronized despite a misaligned polarization configuration. Such states have been observed experimentally in VCSEL arrays [14]. Moreover, polarization dynamics play an important role in the synchronization of master-slave VCSEL configurations, and polarization encoding has recently been proposed for high bit-rate encryption in optical communications [15].

In this Rapid Communication, we develop an extension of the Kuramoto model as a prototype for the study of the fundamental properties of coupled oscillators described by vector fields in which at least two phases play a critical role: One associated with the natural oscillation frequency as in the Kuramoto model, and the other with the direction of oscillation (polarization). We study the synchronization properties of an ensemble of globally coupled non-identical oscillators and show the existence of two transitions: phase synchronization and polarization direction ordering. We develop a self-consistent theory to determine the thresholds for both transitions and show that polarization ordering can never take place if the system is not already synchronized in frequency.

Our analysis is made in the context of a general model, the Vector Complex Ginzburg-Landau Equation (VCGLE), which has been used for modeling different physical systems, from two-components Bose condensates [16] to nonlinear optics [17] including laser emission from wide aperture resonators such as VCSELs $[13,18]$. The VCGLE can be written on symmetry grounds, but the determination of the parameters in the equation requires a specific physical model. We consider here parameter ranges of interest in optics. A set of $N$ globally coupled space-independent VCGLEs is given by

$$
\begin{aligned}
\partial_{t} A_{j}^{ \pm}= & \left(\mu_{j}+i \omega_{j}\right) A_{j}^{ \pm}-\left(1+i \beta_{j}\right)\left(\left|A_{j}^{ \pm}\right|^{2}+\gamma_{j}\left|A_{j}^{\mp}\right|^{2}\right) A_{j}^{ \pm} \\
& -\left(\gamma_{a}+i \gamma_{p}\right) A_{j}^{\mp} e^{ \pm i \delta_{j}}+\frac{C}{N} \sum_{k=1}^{N} A_{k}^{ \pm},
\end{aligned}
$$

where $A_{j}^{+}\left(A_{j}^{-}\right)$is the circularly right (left) polarized compo- 
nent of the $j_{t h}(j=1 \ldots N)$ vector variable, $\omega_{j}$ is the natural oscillation frequency, $\beta_{j}$ gives a nonlinear frequency shift and $\gamma_{j}$ (a real number for lasers) couples the polarization components. The term $\left(\gamma_{a}+i \gamma_{p}\right) \exp \left( \pm i \delta_{j}\right)$ represents an external forcing [18] that linearly couples $A_{j}^{+}$and $A_{j}^{-}$. For example, for a VCSEL, the forcing arises from device anisotropies (dichroism and birefringence) [12] that couple the circularly polarized components of the electric field, introducing two preferential polarization directions. Another example is a ring laser where any localized change in the refraction index breaks the invariance along the ring, introducing the same coupling between the two counterpropagating modes [19] and setting a preferential phase relationship between them. $C$ is the strength of the global coupling which in laser arrays may be induced by external reflections (e.g., by placing a reflection at the common reflector of the array [20]) or by a common active medium [14]. We introduce $A_{j}^{ \pm}=Q_{j}^{ \pm} \exp \left(i \varphi_{j}^{ \pm}\right)$. We consider $\gamma_{j}<1$, for which linearly polarized states $\left(Q_{j}^{+}=Q_{j}^{-}\right)$are stable solutions of the solitary oscillators [13], as is the case of VCSELs [12]. Close to these solutions, we neglect the dynamics for each polarization component amplitude $\left(\dot{Q}_{j}^{ \pm}=0\right)$, so that the system (1) can be described in terms of phase equations for each oscillator: The global phase $\phi_{j}=\left(\varphi_{j}^{+}+\varphi_{j}^{-}\right) / 2$, and the rotational phase $\psi_{j}=\left(\varphi_{j}^{+}-\varphi_{j}^{-}\right) / 2$, whereas the latter determines the linear polarization direction. We have

$$
\begin{gathered}
\dot{\psi}_{j}=\gamma_{a} \sin \left(2 \psi_{j}-\delta_{j}\right)+\frac{C}{N} \sum_{k=1}^{N} \sin \left(\psi_{k}-\psi_{j}\right) \cos \left(\phi_{k}-\phi_{j}\right), \\
\dot{\phi}_{j}=\omega_{j}+\gamma_{p} \cos \left(2 \psi_{j}-\delta_{j}\right)+\frac{C}{N} \sum_{k=1}^{N} \sin \left(\phi_{k}-\phi_{j}\right) \cos \left(\psi_{k}-\psi_{j}\right) .
\end{gathered}
$$

In the uncoupled case $(C=0)$, the global phases $\phi_{j}$ rotate at a constant frequency, whereas the polarization angles $\psi_{j}$ reach a steady state, thus modeling a solitary laser emission. In fact, for $C=0$ we have two orthogonal linearly polarized solutions for the $j_{t h}$ oscillator: $2 \psi_{j}=\delta_{j}, \phi_{j}=\phi_{0 j}+\left(\omega_{j}+\gamma_{p}\right) t$, and $2 \psi_{j}=\delta_{j}+\pi, \phi_{j}=\phi_{0 j}+\left(\omega_{j}-\gamma_{p}\right) t$, where $\phi_{0 j}$ is a constant. For $\gamma_{a}<0$ the first solution is selected, whereas for $\gamma_{a}>0$ the second is selected. In laser physics, the parameter $\gamma_{a}$ models the different linear gain encountered by the two linearly polarized solutions, thus making linearly stable the solution with the higher linear gain. In the same context, the parameter $\gamma_{p}$ models the cavity birefringence [12], which splits the emission frequency of the two orthogonal linearly polarized solutions by an amount equal to $2 \gamma_{p}$. In the following, we take $\gamma_{a}<0$, so we will refer to $2 \psi_{j}=\delta_{j}$ as the natural polarization angle of each oscillator. Our results, however, do not depend on this choice, or on the sign of $\gamma_{p}$, which we set positive. Fixing the polarization degree of freedom ( $2 \psi_{j}(t)=\delta_{j}=\delta_{0}$ for all $j$ ) Eq. (3) reverts to the Kuramoto model: $\dot{\phi}_{j}=\omega_{j}+\gamma_{p}+(C / N) \sum_{k=1}^{N} \sin \left(\phi_{k}-\phi_{j}\right)$.

The differences in the natural polarization angles and frequencies of the oscillators represent two sources of disorder in our system. They are statistical quantities, randomly chosen from two symmetric unimodal distributions $q(\delta)$ and $p(\omega)$, with zero mean and standard deviation $\sigma_{\omega}$ and $\sigma_{\delta}$, respectively. Therefore, we introduce two order parameters to characterize the degree of polarization ordering and phase synchronization, respectively,

$$
\begin{aligned}
& \eta \exp (i \chi)=\frac{1}{N} \sum_{k=1}^{N} \exp \left(i \psi_{k}\right), \\
& \rho \exp (i \theta)=\frac{1}{N} \sum_{k=1}^{N} \exp \left(i \phi_{k}\right) .
\end{aligned}
$$

Without coupling $\rho$ averages to zero while, as $2 \psi_{j}=\delta_{j}, \eta$ accounts for the natural disorder in the polarization angle. In the continuum limit, $\eta=\eta_{0}=\left|\int_{-\pi}^{\pi} \exp (i \delta / 2) q(\delta) d \delta\right|$, which is non-zero unless $q(\delta)$ is a uniform distribution between $-\pi$ and $\pi$.

For small coupling the global phases $\phi_{j}$ are desynchronized, which leads the coupling term in the polarization Eq. (2) to the vanish. Therefore each oscillator remains oscillating in its natural polarization angle. No polarization interaction takes place until the phases $\phi_{j}$ start to synchronize. Increasing $C$, two different scenarios toward polarization ordering and phase synchrony $(\eta=\rho=1)$ are found depending on the relative strength of the polarization $\sigma_{\delta}$ and phase disorder $\sigma_{\omega}$.

For $\sigma_{\omega} \ll \sigma_{\delta}$, the transitions to phase and polarization synchrony are well separated. The phases $\phi_{j}$ synchronize first. The transition to phase synchrony can be analyzed by taking $2 \psi_{j}=\delta_{j}$ (frozen polarizations), so that the set (2) and (3) can be approximated by

$$
\dot{\phi}_{j}=\omega_{j}+\gamma_{p}+\frac{C}{N} \sum_{k=1}^{N} \sin \left(\phi_{k}-\phi_{j}\right) \cos \left(\frac{\delta_{k}-\delta_{j}}{2}\right) \text {. }
$$

Averaging the polarization angles, reduces Eq. (6) to a Kuramoto-like model with an effective coupling $\widetilde{C}$,

$$
\dot{\phi}_{j}=\omega_{j}+\gamma_{p}+\frac{\widetilde{C}}{N} \sum_{k=1}^{N} \sin \left(\phi_{k}-\phi_{j}\right)
$$

where $\widetilde{C}=C \int \cos \left[\left(\delta-\delta^{\prime}\right) / 2\right] q(\delta) q\left(\delta^{\prime}\right) d \delta d \delta^{\prime}=C \eta_{0}^{2}$. The polarization disorder makes the phase coupling less effective but not vanishing. Following the standard treatment of the Kuramoto model [3], the self-consistent equation for the order parameter amplitude $\rho$ reads

$$
\rho=\widetilde{C} \rho \int_{-\pi / 2}^{\pi / 2} \cos ^{2}(\phi) p(\tilde{C} \rho \sin (\phi)) d \phi
$$

Therefore, the critical coupling $C_{t}$ for the onset of collective phase synchronization reads 

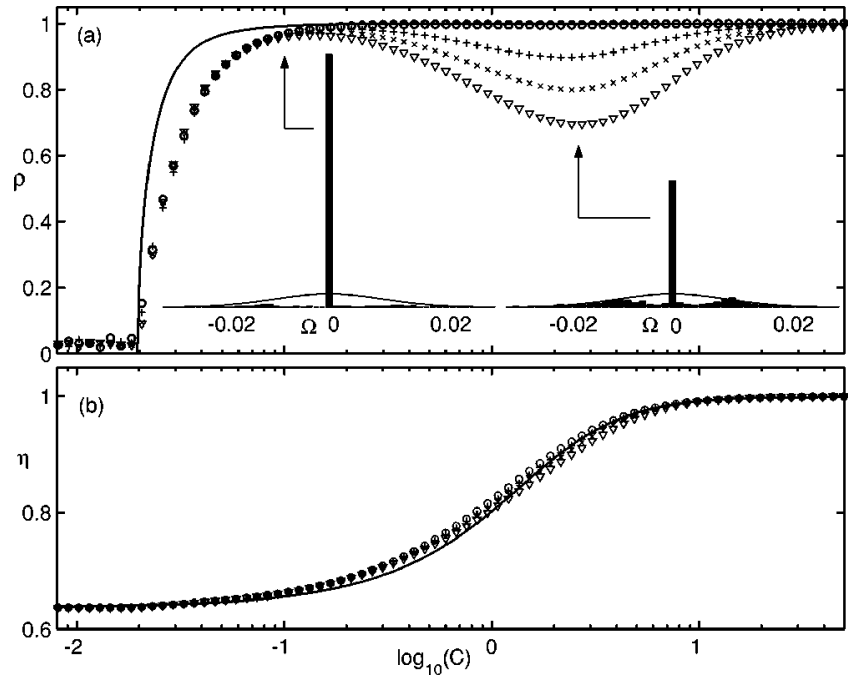

FIG. 1. Amplitude of order parameters $\rho$ and $\eta$ as function of the coupling $C$. The solid line corresponds to the theoretical predictions, whereas the symbols to numerical integration of (2) and (3) for typical VCSEL birefringence values $\left(\bigcirc: \gamma_{p}=0,+: \gamma_{p}=1, \times\right.$ : $\left.\gamma_{p}=2.5, \triangle: \gamma_{p}=5\right)$. We have considered $\gamma_{a}=-0.5, N=10^{3}$, a Gaussian distribution $p(\omega)$ for the natural frequencies with $\sigma_{\omega}=10^{-2}$, and a uniform distribution $q(\delta)=1 / 2 \Delta$ for $-\Delta \leqslant \delta \leqslant \Delta$, with $\Delta=\pi / 2$ $\left(\sigma_{\delta}=\Delta / \sqrt{3}=0.9068\right)$ for the natural polarization angles. The insets show the time-averaged dressed frequencies distribution for $\gamma_{p}=5$, $C=0.1$ (left) and $C=2.5$ (right). The natural frequency distribution $p(\omega)$ is shown for reference (solid line).

$$
C_{t}=\frac{2}{\pi p(0) \int \cos \left[\left(\delta-\delta^{\prime}\right) / 2\right] q(\delta) q\left(\delta^{\prime}\right) d \delta d \delta^{\prime}}
$$

Figure 1 shows the good agreement between the transition to phase synchronization obtained from numerical integration of (2) and (3) and the solution of the self-consistent Eq. (8). Notice the excellent agreement obtained for the onset of synchronization given by, (9) $C_{t}=0.01968$. The distribution of averaged dressed frequencies $\Omega=\langle\dot{\phi}\rangle$ (left inset of Fig. 1) shows a highly dominant peak which comes from the synchronized oscillators $(0.97 N$ in this case $)$. Notice also that for $C<C_{t}$ the polarization order parameter takes a constant value $\eta_{0}$ which corresponds to the initial polarization disorder, in agreement with the assumptions leading to (7).

Increasing further the coupling strength, the oscillators leave the respective natural polarization angles and start to order in polarization. As the phase synchronization has already been achieved, we are now in the position to develop a self-consistent theory for the polarization ordering as follows: Assuming perfect phase synchronization $\left(\phi_{k}=\phi_{j}\right)$, Eq. (2) becomes

$$
\dot{\psi}_{j}=\gamma_{a} \sin \left(2 \psi_{j}-\delta_{j}\right)+\frac{C}{N} \sum_{k=1}^{N} \sin \left(\psi_{k}-\psi_{j}\right) .
$$

Since the individual polarization is not self-oscillating, Eq. (10) is not a Kuramoto-like model. However, from (4) we have $\eta \sin \left(\chi-\psi_{j}\right)=(1 / N) \sum_{k=1}^{N} \sin \left(\psi_{k}-\psi_{j}\right)$, which introduced in Eq. (10) yields

$$
\dot{\psi}_{j}=\gamma_{a} \sin \left(2 \psi_{j}-\delta_{j}\right)-C \eta \sin \left(\psi_{j}-\chi\right) .
$$

The stationary solution $\bar{\psi}_{j}(\delta, \eta, \chi)$, given implicitly by

$$
\gamma_{a} \sin \left(2 \bar{\psi}_{j}-\delta_{j}\right)-C \eta \sin \left(\bar{\psi}_{j}-\chi\right)=0,
$$

can be introduced in Eq. (4) to self-consistently find $\eta$ and $\chi$. In the continuum limit we have

$$
\eta \exp (i \chi)=\int \exp [i \bar{\psi}(\delta, \eta, \chi)] q(\delta) d \delta .
$$

Altogether, Eqs. (12) and (13) allow for the calculation of the polarization order parameter, for example through a NewtonRaphson method, so that the polarization ordering can be fully described. The imaginary part of integral (13) was found to vanish $(\chi=0)$, if $q(\delta)$ is even. Figure 1 shows the agreement between the evaluation of $\eta$ using the definition (4) with the results of the numerical integration of the full set Eqs. (2) and (3) and using the self-consistent approximation given by Eqs. (12) and (13). We obtain good results even for small coupling where global phases are desynchronized (in that regime the contribution of the coupling term in the polarization equation is negligible). The ordering of the polarization directions induces a loss of coherence where the phases partially de-synchronize, lowering $\rho$. The reason is that, as the polarization order is increased, the polarization angles depart from the natural angle, and therefore the term $\gamma_{p} \cos \left(2 \psi_{j}-\delta_{j}\right)$ in Eq. (3) plays the role of an added disorder to the natural frequencies $\omega_{j}$. Increasing $\gamma_{p}$, this effect is linearly increased, enhancing the coherence loss extent, as shown in Fig. 1. For $\gamma_{p}=5, \rho$ is reduced down to 0.65 . The averaged dressed frequency distribution (right inset of Fig. 1) shows that the peak at $\Omega=0$ is lowered in the same proportion and two lateral lobes associated with drifting oscillators appear, yielding an overall shape for the distribution similar to that of partially synchronized Kuramoto oscillators [3]. From a practical point of view, the coherence lowering would have a direct impact the output intensity in VCSEL arrays. A reduction of $\rho$ down to 0.65 leads to coherent output intensity of only $40 \%$ with respect to the fully synchronized case. Finally, for large coupling, complete phase synchronization and polarization ordering are achieved.

Numerical simulations for different values of $\sigma_{\delta}$ showed that decreasing the disorder in the natural polarization angles, the polarization transition to synchronization shifts to lower values of the coupling. However, a polarization order enhancement is not possible before the phases start to synchronize, so for $\sigma_{\omega} \approx \sigma_{\delta}$ or $\sigma_{\omega}>\sigma_{\delta}$ the two transitions take place simultaneously. Nevertheless, the polarizations are still effectively uncoupled until the phases start to synchronize, so the self-consistent Equation (8) still holds as well as the prediction (9) for the phase synchronization onset $C_{t}$, which now also signals the onset of the polarization ordering as shown in Fig. 2. The self-consistent equation for the polarization order parameter still gives a good description of the polarization order enhancement. 


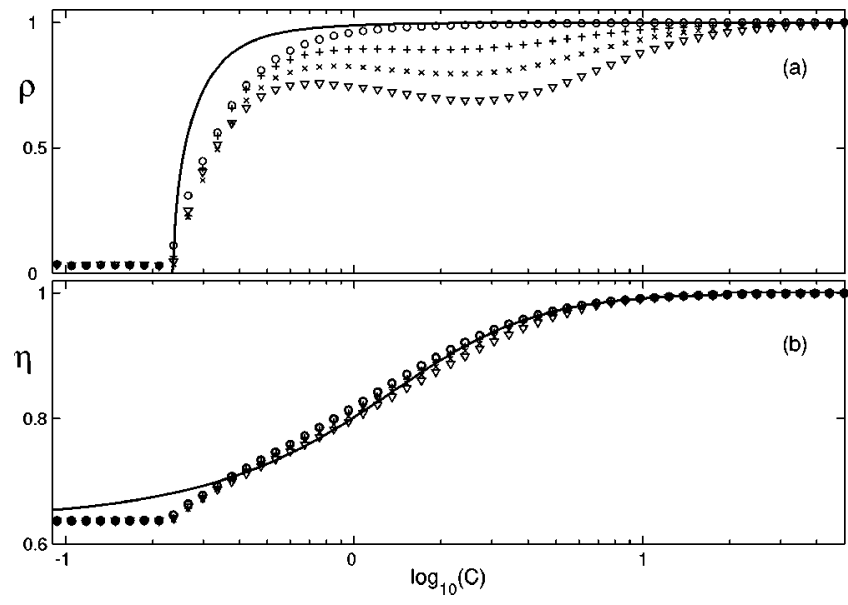

FIG. 2. Amplitude of order parameters $\rho$ and $\eta$ as in Fig. 1, but with larger natural frequency disorder $\sigma_{\omega}=0.12$, (now $C_{t}=0.2362$ ).

In conclusion, we have introduced a theoretical framework to study the synchronization properties of a system of globally coupled oscillators extending the results for limit cycle oscillators to include the consideration of oscillation direction (polarization). Two sources of disorder are included: Randomly distributed natural frequencies and natural oscillation directions. Increasing the coupling no polarization order enhancement is possible until the phases start to synchronize, because the phase disorder destroys the interaction among the polarization variables. This is in agreement with experimental results observed in VCSEL arrays [14]. Typically, the frequencies synchronize first, and polarization synchrony takes place at a higher coupling level, through a partial de-synchronization of the phases (coherence lowering). We have developed self-consistent approximations which provide a very good estimate of the synchronization properties of system. Increasing the disorder in the natural frequencies or decreasing the disorder in the natural polarization angle the two transitions merge in a unique process to full synchrony, and we provided the critical coupling for its onset.

This work has been funded by the European Commission through VISTA HPRN-CT-2000-00034, the Spanish MCyT under project BFM2000-1108, MCyT and Feder SINFIBIO BFM 2001-0341-C02-02.
[1] S. H. Strogatz, Nature (London) 410, 268 (2001); A. Pikovsky, M. Rosenblum, and J. Kurths, Synchronization (Cambridge University Press, Cambridge, UK, 2001).

[2] A. T. Winfree, J. Theor. Biol. 16, 158 (1967); The Geometry of Biological Time (Springer, New York, 1980).

[3] Y. Kuramoto, in Proceedings of the International Symposium on Mathematical Problems in Theoretical Physics, edited by H. Araki, Lecture Notes in Physics, Vol. 39 (Springer, Berlin, 1975); Chemical Oscillations, Waves and Turbulence (Springer, Berlin, 1984); S. H. Strogatz, Physica D 143, 1 (2000).

[4] C. S. Peskin, Mathematical Aspects of Heart Physiology (Courant Institute of Mathematical, Science, New York, 1975).

[5] K. Wiesenfeld, P. Colet, and S. H. Strogatz, Phys. Rev. Lett. 76, 404 (1996); Phys. Rev. E 57, 1563 (1998).

[6] J. A. Acebron and R. Spigler, Phys. Rev. Lett. 81, 2229 (1998).

[7] L. Fabiny, P. Colet, R. Roy, and D. Lenstra, Phys. Rev. A 47, 4287 (1993). A. Hardy and E. Kapon, IEEE J. Quantum Electron. 32, 966 (1996).

[8] H. Pier, E. Kapon, and M. Moser, Nature (London) 407, 880 (2000).

[9] H. G. Winful and L. Rahman, Phys. Rev. Lett. 65, 1575 (1990); H. G. Winful, Phys. Rev. A 46, 6093 (1992); S. Riyopoulos, ibid. 66, 053820 (2002).
[10] G. Kozyreff, A. G. Vladimirov, and P. Mandel, Phys. Rev. Lett. 85, 3809 (2000).

[11] D. Botez and D. R. Scifres, Diode Laser Arrays (Cambridge Univ. Press, Cambridge, UK, 1994).

[12] M. San Miguel, in Semiconductor Quantum Optoelectronics: from Quantum Physics to Smart Devices, edited by A. Miller, M. Ebrahimzadeh, and D. M. Finlaynson (IOP, Bristol, 1999).

[13] M. San Miguel, Phys. Rev. Lett. 75, 425 (1995); E. Hernández-García, M. Hoyuelos, P. Colet, and M. San Miguel, ibid. 85, 744 (2000).

[14] P. Debernardi, G. P. Bava, F. Monti di Sopra, and M. B. Willemsen, IEEE J. Quantum Electron. 39, 109 (2003).

[15] A. Scirè, J. Mulet, C. R. Mirasso, J. Danckaert, and M. San Miguel, Phys. Rev. Lett. 90, 113901 (2003).

[16] R. Graham and D. Walls, Phys. Rev. A 57, 484 (1998).

[17] M. van Hecke, C. Storm, and W. van Saarlos, Physica D 134, 1 (1999).

[18] A. Amengual, D. Walgraef, M. San Miguel, and E. HernándezGarcía, Phys. Rev. Lett. 76, 1956 (1996).

[19] M. Sorel, P. J.R. Laybourn, A. Scirè, S. Balle, R. Miglierina, G. Giuliani, and S. Donati, Opt. Lett. 27, 1992 (2002); R. J. C. Spreeuw, M. W. Beijersbergen, and J. P. Woerdman, Phys. Rev. A 45, 1213 (1992).

[20] S. Y. Kourtchatov, V. V. Likhanskii, A. P. Napartovich, F. T. Arecchi, and A. Lapucci, Phys. Rev. A 52, 4089 (1995). 